\title{
A Method of Organization Planning in Scientific Research Task Based-on Role
}

\author{
Shaohua Wang ${ }^{1,2} *$ \\ 1, Faculty of Management and Economics, \\ Dalian University of Technology, \\ Dalian, China; \\ 2, Dept. of Scientific Research, \\ Dalian Naval Academy, Dalian , China \\ songzywb@hotmail.com \\ * Corresponding Author
}

\author{
Lili Rong \\ Faculty of Management and Economics, \\ Dalian University of Technology, \\ Dalian, China \\ sh2680025@126.com
}

\begin{abstract}
A method of organization planning in scientific research task based-on role is presented to content the needs of increasing abilities of task assigning and efficiency of functioning and lessening proportions of recourse deploying. A mode of scientific research organization based-on role is established, which is used to describe and restrict the entities taking on scientific researching tasks with the social properties of abilities, authorities and obligations. With this mode, a matching method of scientific research resources based-on role and task is presented, through which managing tasks and resources based on role properties are used to improve the efficiency of task implementing in scientific research.
\end{abstract}

Keywords-Scientific Research Management; Role; Organization

\section{INTRODUCTION}

Scientific research institutes, as forefronts of units for scientific research and technological innovation, science and technology information manufacture and production have many scientific research project management, which cover wide areas and large quantities of majors, professions and disciplines. Current scientific research management in the form of organization and structure are almost consistent with the traditional way, but with the development of modern management theory and information technology, scientific research management and organization are evolving from the simple, tree structure form to tree-stemmed reticular direction structure, which has both higher and lower task allocation and assignment, and at close collaboration between the same level, and even between hierarchical task collaboration. Task oriented, function-based organization is the main form of scientific research organization under the situation of informatization. Current research projects generally operate in the form of the project teams in interdisciplinary and interdisciplinary problems, bringing challenges for scientific research management. These management problems are essentially involved in allocation, matching and optimization problems between the main body of scientific research and resource. Organization of scientific research projects (team) carry on the design and planning, project management in the process of task assignment, functional use, resource allocation and other planning and research, in order to improve the efficiency and reduce costs. This is also a need to be solved in current scientific research project management is one of the major problems.

This article is aimed at analyzing these problems that exist in the current scientific research management. It puts forward the planning method based on the role of scientific research organization, introduces the role concept of general social conduct into the field of scientific research management, and establishes the scientific research organization description model based on role. The mode uses the roles, abilities, rights and obligations and other social attributes to describing and constraining scientific research entities (individuals, teams, organizations) undertaking the tasks and to establish a matching method based on task-role of scientific research resources. The mode can fusion the relationship between research organizations (or team) and the one between individuals and organizations, use the attributes for the role of scheduling tasks and resources to improve the efficiency of scientific research tasks.

\section{STATUS ANALYSISOF ORGANIZATIONPLANNING OF RESEARCH T ASKS}

From the macro level, research planning and management includes scientific research comprehensive planning departments, scientific research plan management, business department, scientific research units and users. Scientific research plan, in the execution and implementation process, generally includes "planning requirements planning stage", "task planning stage", "scientific research project implementation stage" and "the stage of transformation of scientific and technological achievements". In the scientific research project management, scientific research organizations include at least the following entities, such as, decision-making team, management team, research and development team, the domain experts, security team and users, etc. Scientific research project development process, including requirements analysis, design, difficult points (key technology) research, technology development, tes $\mathrm{t}$ and evaluation, backtracking, delivery (completion) and so on. In the project management and the scientific research project management of scientific research, the current main mode of completing the above scientific research 
task lies in the form of scientific research organization or in the form team for project management and development, that is, the project team is one of the main objects of scientific research management, and undertakes the main body of the project research and development. Fully using the modern theory of organization design to run the project organization design and project management is the main form of modern scientific research project management [1].

The design of organization structure and organization operation system is the main content of the modern project organization design [2]. Among numerous studies, the organizational design methodology based on the organization theory of calculation has been one of the widely recognized contents as part of the research, and it has become an important research direction [1][3-7].The theory has provided a new way and method for commercial organizations and public management organizations, military organizations, and other informal organizations and other social entity organization behavior and process studies, and due to the scale and level of the complex structural research, it is difficult to solve the problems, such as the earth's climate, the analysis of the problems such as the human genome.

Scientific research project management and operation is a typical social entity organization. The purpose of adopting the project organization design based on calculation of organization theory and management is to improve the management efficiency, and find the effective ways of reducing resource utilization. Although considering fro $m$ the computational angle, the organization can design a suitable mode for the design of scientific research project management organization and operation mode. It is often overlooked how the social nature of scientific research organization such as the power of the organization, obligations, social norms will reflect in organization design and operation management. Introducing the concept of "role", this paper combines "social role" with "calculation", designs and plans for the organization of scientific research management. The definition of role is firstly presented.

\section{THE ORGANIZATION'S ROLE MODEL}

\section{A. role's definitions}

According to the role of social attribute, namely, the role is a set of rights, standardized obligations and behavior patterns, which is consistent with the status of the individual in a group. It is also the expectation of a certain individual's behavior in a particular position, being the basis of a group [8]. As a kind of abstract logic level, the role has realized the isolation between individuals so as to make a more stable composition of the group and more flexible and reliable allocation and implementation of the tasks.

To make clear the definition of the role in practical work, the relevant definition of the organization is given first.

The first organization related concept definition is given. Here organization (Organizations) is defined by a four-concept group:

Organization: $=\langle\mathrm{ID}$, Entities, Tasks, Goals $>$

The Organization is defined as the combination of the entity, a collection of tasks and goals. Among them,
(1) Organization identifier (ID). It is used to identify the organization's position in the scientific research management behavior;

(2) Entity (can be marked as EntitiesQ). It refers to the participants in the plan or task undertaken by the organization. It can be either the individual or the lower organization. Q is marked as the mutual relationship.

(3) Tasks represent the assemblies of the organization tasks at the corresponding level. Goals are given from the corresponding level to the lower level. Tasks include the target description of organization at the corresponding level, namely Goals Tasks 。

Organization action is restrained by the common plans in internal organization, which provides objective constrains for the action plan of the external organization. Its plans and tasks should be worked out by the corresponding level organization.

Considering organization from the point of view of sociology, this paper organizes the role definition:

The role in the organization refers to the combination of behavior norms and behavior patterns which are consistent with their position in the organization. It is the expectation of a certain behavior with specific capability. In other words, the role is the status of the entity in the origination. It is also an action to obey and accomplish the orders and plans from the organization with its inherent abilities. Role specifies the action range of the entity in the organization and the corresponding obligations and behavior norms corresponding to its status.

According to the role definition, role in the organization should have the following features:

(1) The role has its own properties and ability.

(2) The role has the organizational characteristics, namely the position occupied by the entity in the organization. Entity is always in the form of individual, group or organization, combined through some relationship between each other. Entities need to understand their place in the team, the relationship between their superiors, subordinates, neighbors and so on. Its team attribute should include a description of the organization characteristics.

(3) Role should undertake its responsibility corresponding to its capacity and status (position). In other words, it should have social attribute, which belongs to a part of the behavioral model in the organization. First, the entity first shall know its ability and position in the organization, then take responsibility fairly, even if sometimes the duty or task plan is beyond the scope of its capacity. Sometimes for the operational behavior, the organization needs to undertake unconditionally.

(4) Role in the organization has the characteristics of being dependent, dynamic and diverse [8]. Dependence refers to the role of position depending on its ability and position in the organization, its existence depending on the mission or command; Dynamics refers to the fact that adding and deleting role can be realized in the organization life cycle according to its needs. Diversity refers to the fact that an entity can play different roles. Because the process is dynamic and uncertain, the organizational structure will change along with the advancement of process, as well as the entities' join and leave. The change of the role is dynamic, which needs time-related process to depict. Its content should include reason, process and result, etc. 


\section{Among them,}

(1) Entities act as the role of entity and can show static relationship with other team or organization between individuals

(2) Abilities refer to the behavior in the organization to complete a task and that behavior can achieve the goal of the task.

(3) Authorities refer to the power corresponding to its abilities and status, including the jurisdiction of the use of resources and operation actions, etc. Authorities should be established by the rules.

(4) Politics restrains the role legally. It defines the position of role, corresponding duties, obligations and the authorities of allocating resources. Rules can be used to limit the role by the following two ways:

Only provides rules which must be fulfilled. In this way, roles only prescribe responsibilities and obligations which must be fulfilled. Role with corresponding abilities and status should perform such rules.

Only provides rules which should not be violated. In this way, politics only describe rules which should not be violated.

Combine the above-mentioned two ways to limit the entity's position and authorities.

(5) Goals, power and status are the static attributes of role. Role can be truly realized only when targets are given to the entity or organization.

The objectives (Goals), power and status is the role of static attributes, only when given entity or organization in order to target, role can truly reflect.

\section{B. Principle of Role-Task Construction}

Taking realization of a task into consideration, researchers must divide each stage of the task into some atomic tasks, which can be directly fulfilled by entities. Only in this way can the roles be implemented in the task. When doing task division and role assignment, researchers have to refer to the following rules:

(1) Principle of task-divisibility: Any tasks are dividable, that is to say any tasks can be divided into a series of atomic-task sets, and the breakdown is nonunique.

(2) Principle of atomic-task existence: There exists at least one atomic task which matches the divided task.

(3) Princip le of atomic-task limitation: The ato mic-task sets of the divided task are bounded and finite.

(4) Principle of multi-role acting: A role can be taken on by a number of different entities.

(5) Principle of single-role: An atomic task can only be taken on by one role.

(6) Principle of single-task: A role can handle mu ltiple atomic tasks, but it can only handle one simultaneously.

Principles (1) (3) explain that tasks are finite and dividable; Principle (4) set a rule for the physical properties of roles; Principles (5) and (6) establish the corresponding relations between roles and tasks. Based on the principle of role-task construction, researchers can divide a task into atomic task sets that can be calculated.

\section{RESOURCE AlLOCATION BASED ON ROLES}

\section{A. Basic Methods of Allocating Resources}

In scientific research project management, all hard and soft environments that can support the smooth implementation and operation of the project can be called resources in a broad sense. Hard environment includes site, funds, etc., while soft environment contains regulations, agreements, etc. In scientific research management, the resource allocation based on roles should refer to the following steps:

(1) By dividing the task, researchers can form atomic task set TM, resource demand VP and order relationship between tasks TR. Here, researchers have $\mathrm{TM}=\left\{t_{1}\right.$, $\left.t_{2}, \ldots, t_{m}\right\} . \mathrm{m}$ is the number of atomic tasks which are divided from the original task. TA refers to the resource demand of processing the task $\mathrm{VP}=\left(v_{1}, v_{2} \ldots, v_{p}\right)$, and $\mathrm{v}_{\mathrm{p}}$ is the p-type resource needed for processing the task. TR describes dependencies between tasks qualitatively, such as logical sequence, priorities and concurrent relation of tasks, etc. [2].

(2) Establish a role set $\mathrm{RN}=\left\{r_{1}, r_{2}, \ldots r_{n}\right\}$, where each role ri has an entity $\mathrm{EN}$ to get involved, and also has capability set AN, permission UN and other basic properties. Here, role is replaced by capability to do the AN-TM matching calculation, and form the capabilitytask matrix:

$$
\left.\begin{array}{c}
(1) \\
a_{1} \\
a_{2} \\
\vdots \\
a_{n}
\end{array}\right] \times\left[\begin{array}{llll}
t_{1} & t_{2} & \ldots & t_{m}
\end{array}\right]=\left[\begin{array}{cccc}
a_{1} t_{1} & a_{1} t_{2} & \ldots & a_{1} t_{m} \\
a_{2} t_{1} & a_{2} t_{2} & \ldots & a_{2} t_{m} \\
\vdots & \ldots \ldots & \vdots \\
a_{n} t_{1} & a_{n} t_{2} & \ldots & a_{n} t_{m}
\end{array}\right]
$$

(2) Do the TM-VP matching calculation, thus the task-resource requirements matrix is formed:

$$
\left[\begin{array}{c}
t_{1} \\
t_{2} \\
\vdots \\
t_{m}
\end{array}\right] \times\left[\begin{array}{llll}
v_{1} & v_{2} & \ldots & v_{p}
\end{array}\right]=\left[\begin{array}{cccc}
t_{1} v_{1} & t_{1} v_{2} & \ldots & t_{1} v_{p} \\
t_{2} v_{1} & t_{2} v_{2} & \ldots & t_{2} v_{p} \\
\vdots & \ldots \ldots & \vdots \\
t_{m} v_{1} & t_{m} v_{2} & \ldots & t_{m} v_{p}
\end{array}\right]
$$

(3) Multiply the above matrixes to form the charactercapability-task matrix and the resources-demand matrix, which can be noted as followings:

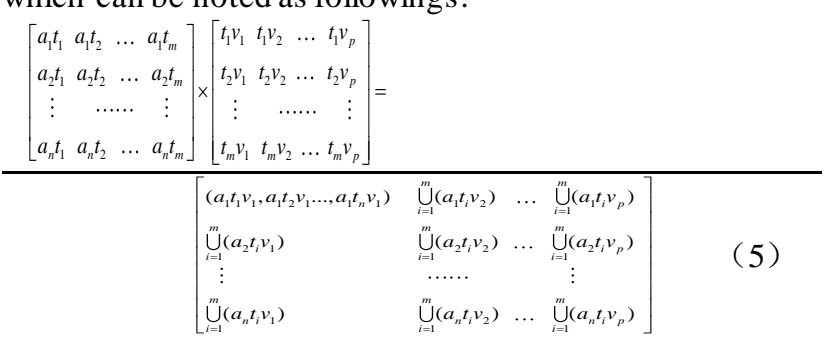

Formula (5) is adjusted and simplified according to two principles: one is that the matrix multiplication is not added, but the union is computed; the other is that role matching of different tasks and recourse allocation cannot be merged, which is antitjvp, $(\mathrm{i} \neq \mathrm{j})$ denoted 0 , and antitjvp, $(i=j)$ denoted by amtivp. The first principle guarantees the completeness of atomic tasks in the plan; while according to different roles, the second principle establishes principle (6) to ensure that unrelated tasks cannot share the same roles and resources simultaneously. Theoretically, there are $\mathrm{n}^{*} \mathrm{~m}^{*} \mathrm{p}$ possible ways to make a role allocate resources and complete the task. 
(4) Check the constraint conditions of permis sions that roles use resource demand. In formula (5), on the right side of the matrix, set all am in amtivp as 0 in condition that they do not match vp. In this way, researchers can remove the resources that are cannot be allocated by a role; in other words, although the role has capability, it still cannot complete the task. If there is a row or a column to be 0 in the matrix, this indicates that there are sub-tasks that cannot be completed, and therefore the planning is invalid, which requires re-dividing of tasks or roles.

(5) Based on the dependencies between tasks described in TR, establish a diagram describes a role completes the task by allocating resources. This diagram is a directed graph about the task.

(6) To complete the task and not to repeat it, researchers make $\mathrm{i}$ fro $\mathrm{m} 1$ to $\mathrm{m}$, and make ti to traverse the matrix. Consider the following conditions:

i) When tasks are in sequential relationship, roles can bear the task accordingly;

ii) When tasks are in parallel relationship, the relationship between roles and tasks can only be one-toone corres pondence.

In this way, researchers can simplify the diagram according to the relationship between tasks, calculate the path of the diagram, and get the roles and resources that are needed to complete the task.

\section{B. instance analysis}

Make the character set $A=\{a 1, a 2, a 3, a 4\}$, task set $\mathrm{T}=\{\mathrm{t} 1, \mathrm{t} 2, \mathrm{t} 3, \mathrm{t} 4, \mathrm{t} 5\}$, and resource set $\mathrm{V}=\{\mathrm{v} 1, \mathrm{v} 2, \mathrm{v} 3, \mathrm{v} 4\}$. Task diagram is shown in Fig. 1. The number of possible divisions may be $4 * 5 * 4=80$.

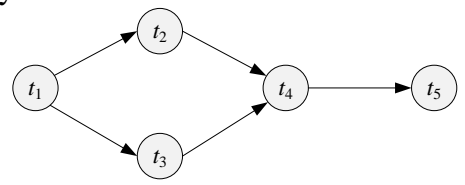

Figure 1. Task diagram

By calculation, researchers have the roles-tasksresource set: $\{\mathrm{a} 1 \mathrm{t} 1 \mathrm{v} 1, \mathrm{a} 2 \mathrm{t} 1 \mathrm{v} 2, \mathrm{a} 2 \mathrm{t} 2 \mathrm{v} 1, \mathrm{a} 3 \mathrm{t} 2 \mathrm{v} 4, \mathrm{a} 2 \mathrm{t} 3 \mathrm{v} 4$, $\mathrm{a} 3 \mathrm{t} 4 \mathrm{v} 2, \mathrm{a} 1 \mathrm{t} 5 \mathrm{v} 3, \mathrm{a} 2 \mathrm{t} 5 \mathrm{v} 1\}$. Th is planning set includes two $\mathrm{t} 1$, two kinds of $t 2$, one $t 3$, one $t 4$, and two $t 5$, so the number of possible divisions is $2 * 2 * 1 * 1 * 2=8$. Planning sets are formed as follows:
(1) $\left\{a_{1} t_{1} v_{1}, a_{2} t_{2} v_{1}, a_{2} t_{3} v_{4}, a_{3} t_{4} v_{2}, a_{1} t_{5} v_{3}\right\}$
(2) $\left\{a_{2} t_{1} v_{2}, a_{3} t_{2} v_{4}, a_{2} t_{3} v_{4}, a_{3} t_{4} v_{2}, a_{2} t_{5} v_{1}\right\}$
(3) $\left\{a_{1} t_{1} v_{1}, a_{2} t_{2} v_{1}, a_{2} t_{3} v_{4}, a_{3} t_{4} v_{2}, a_{1} t_{5} v_{3}\right\}$
(4) $\left\{a_{2} t_{1} v_{2}, a_{3} t_{2} v_{4}, a_{2} t_{3} v_{4}, a_{3} t_{4} v_{2}, a_{2} t_{5} v_{1}\right\}$
(5) $\left\{a_{1} t_{1} v_{1}, a_{2} t_{2} v_{1}, a_{2} t_{3} v_{4}, a_{3} t_{4} v_{2}, a_{1} t_{5} v_{3}\right\}$
(6) $\left\{a_{2} t_{1} v_{2}, a_{3} t_{2} v_{4}, a_{2} t_{3} v_{4}, a_{3} t_{4} v_{2}, a_{2} t_{5} v_{1}\right\}$
(7) $\left\{a_{1} t_{1} v_{1}, a_{2} t_{2} v_{1}, a_{2} t_{3} v_{4}, a_{3} t_{4} v_{2}, a_{1} t_{5} v_{3}\right\}$
(8) $\left\{a_{2} t_{1} v_{2}, a_{3} t_{2} v_{4}, a_{2} t_{3} v_{4}, a_{3} t_{4} v_{2}, a_{2} t_{5} v_{1}\right\}$

Considering the principle that parallel tasks cannot be taken on by the same role, the above mentioned planning which contains both $\{\mathrm{a} 2 \mathrm{t} 2\}$ and $\{\mathrm{a} 2 \mathrm{t} 3\}$ items should be removed. Therefore, (1), (3), (5), (7) in the above planning does not satisfy this condition and should be removed.

In the rest of the planning, researchers can calculate the task amount taken on by a role, as well as the utilization quantity and utilization rate of resources. Determine the allocation scheme of roles and resources according to the demand, and researchers can obtain a scientific research organization design based on the role.

\section{CONCLUSIONS}

In this paper, the organization design method based on the role has been put forward. Combine the calculability of computation organization and sociability of scientific research organization, transform the organizational role, and then researchers can make different organizations (entities) involved in management and development of a scientific research project by taking on different roles. Therefore, the calculation of task intensity and resource utilization becomes much easier. Being a complete organization design method of computation program, it is easy to use computer to calculate and manage, and at the same time provides intuitive results. Therefore, it provides a new way and approach for scientific research organization design.

\section{REFERENCES}

[1] WANG Zhijun, Research on Method of Project Organizational Design Based on Computing Organization Theory[D], Hebei University of Engineering, May, 2008.

[2] Zhou Guijin, Yu yunbo, Design Rules and Changing Trend of Modern Organization[J], Commercial era, 2006(26):42 44.

[3] YANG Dong-sheng, LIU Zhong, ZHANG Wei-ming, etc. An Approach to Describe Organizatio[J], System EngineeringTheory \& Practice, 2004.3(3): 1-7.

[4] K. M. Carley, M. J. Prietula. Computational Organization Theory[J]. Lawrence Erlbaum, Associates Hillsdale NJ, 1994.

[5] Georgiy M. Levchuk, Yuri N. Levchuk, Jie Luo, etc., Normative Design of Organization-Part I: Mission Planning[J], IEEE TRANSCATION ON SYTEM, MAN, AND CYBERNETICSPART A: SYSTEM AND HUMANS, VOL 32, NO.3, MAY 2002: 346 359.

[6] Georgiy M. Levchuk, Yuri N. Levchuk, Jie Luo, etc., Normative Design of Organizations-Part I: Mission Planning[J], IEEE TRANSCATION ON SYTEM, MAN, AND CYBERNETICSPART A: SYSTEM AND HUMANS, VOL 32, NO.3, MAY 2002: 346 359.

[7] Georgiy M. Levchuk, Yuri N. Levchuk, Krishna R. Pattipati, etc., Normative Design of Project-Based Organizations-Part III: Modeling Congruent Robust, and Adaptive Organizations [J], IEEE TRANSCATION ON SYTEM, MAN, AND CYBERNETICS - PART A: SYSTEM AND HUMANS, VOL 34, NO.3, MAY 2004: 337 350.

[8] ZHU Jun, TANG Yong, Collaborative Research on Hierarchical Awareness Model for Role-Based Group Collaborative Journal of Soft ware, Vol.18, Supplement, December 2007:95 101.

[9] VanDick,R,U.Wagner, Categroy salience and organizational identification[J], Journal of Occupational and Organizational Psychology, 2005: 273 285.

[10] Xiong Houren, Chen Xingyuan, Zhang Bin, etc., Scalable Access Control Model Based on Double-tier Role and Organization[J], Journal of Electronics \& Information Technology, 2015(7):1612 1619 